\title{
Length-weight relationship of cartilaginous fish species from Central Aegean Sea (Izmir Bay and Sığacık Bay)
}

\section{Orta Ege Denizi'ndeki (İzmir Körfezi ve Sığacık Körfezi) kıkırdaklı balıkların boy-ağırlık iliş̧kisi}

\section{Elizabeth Grace Tunka Eronat* • Okan Özaydın}

Ege University, Faculty of Fisheries, 35100, Bornova-Izmir, Turkey

${ }^{*}$ Corresponding author: tunkaeronat@hotmail.com

How to cite this paper:

Eronat, E.G.T., Özaydın, O., 2014. Length-weight relationship of cartilaginous fish species from Central Aegean Sea (Izmir Bay and Sığacık Bay). Ege J Fish

Aqua Sci 31(3): 119-125. doi: 10.12714/egejfas.2014.31.3.01

Özet: Bu çalışmada Orta Ege Denizi'nden yakalanmış 30 kıkırdakıı balık türünün (11 köpekbalığı, 18 vatoz ve 1 Chimaera) 16'sının boy-ağırlık ilişkisi incelenmiş ve sunulmuştur. Örnekler bir araşııma gemisi ve bir ticari trol teknesi ile $<500 \mathrm{~m}$ derinlikten 2008-2009 yılları arasında yakalanmıştı. Boy-ağırlık ilişkisi parametrelerinden b değeri 2.79 (Torpedo marmorata) ile 3.78 (Scyliorhinus stellaris), a değerleri 0.0002 (Scyliorhinus stellaris) ile 0.9713 (Dasyatis pastinaca).arasında değişmiştir. Bu çalışma doğu Akdeniz'de yapılmış çalışmalar arasında en yüksek tür sayııını elde etmiş ve incelemiştir.

Anahtar kelimeler: Boy-ağırlık ilişkisi, Kıkırdakı balıklar, İzmir Körfezi, Sığacık Körfezi, Orta Ege Denizi.

Abstract: In this study, length-weight relationship parameters of 16 out of 30 caught cartilaginous fish species examined (11 Sharks, 18 Batoids and 1 Chimaera) from the Central Aegean Sea and presented. Samples were caught from depths of $<500 \mathrm{~m}$ by research vessel and a commercial trawler, between 2008-2009. The values of the slope $b$ in the length-weight relationship parameters ranged from 2.79 (Torpedo marmorata) to 3.78 (Scyliorhinus stellaris), a values from 0.0002 (Scyliorhinus stellaris) to 0.9713 (Dasyatis pastinaca). This study has optained and examined the most chondrichthyan species among the studies in the eastern Mediterranean.

Keywords: Length-weight relationship, Cartilaginous fishes, Izmir Bay, Sığacık Bay, Central Aegean Sea.

\section{INTRODUCTION}

Cartilaginous fishes (Chondrichthyes) in Turkish seas, corresponds to $75 \%$ of the chondrichthyes fauna in Mediterranean (Bradai et al. 2012) and $13 \%$ of the fish fauna of Turkey with 66 cartilaginous species and almost all (61 species) inhabits in the Aegean Sea (Bilecenoğlu et al., 2014). Even though Aegean Sea has the most diverse cartilaginous fish fauna after Turkish waters of Mediterranean due its unique oceanographic (physical, biological and chemical) features, unfortunately there is not enough information concerning their ecological role in the trophic chain or their biology and the knowledge; ones we have are mostly on a few species due to their commercial value. Some species that occurs in the area have a commercial value as food resource (such as Mustelus mustelus, Raja clavata) and after being processed exported to other countries or lately fished as an aquarium species (such as Sycliorhinus canicula or Myliobatis aquila) but they are usually bycatch species and are utilized by fish meal factories as an ingredient.
There are relatively few but increasing studies on chondrichthyan species biology in the Turkish waters. The studies are mostly on their morhology (Filiz and Taşkavak, 2006), length-weight relationship (LWR) (Filiz and Mater, 2002; Yeldan and Avsar, 2007) and some on their diets (Yıgın and Işmen, 2010a; Eronat and Ozaydin, 2014) and reproduction biology (Saglam and Ak, 2012). The studies on their LWR main interest is mainly on bony species but there are few studies specifically on chondrichthyans (Filiz and Mater, 2002; Ismen et al. 2009; Yıgın and Ismen, 2008).

Length and weight data are a useful and standard result of fish sampling programs. These data are needed to estimate growth rates, length and age structures, and other components of fish population dynamics (Kolher et al., 1995). It also allows fisheries scientists to convert growth-in-length equations to growth-in-weight for stock assessment models (Pauly, 1993).

The aim of the study is to determine the cartilaginous fish fauna of Central Aegean Sea and to estimate the length and weight parameters for further studies. 


\section{MATERIALS AND METHOD}

The study areas (Izmir Bay and Siğacık Bay) are positioned in central Aegean Sea and have different ecological features due to their geographical positions, bathymetry and human pressure (Figure 1). Where most parts of Izmir Bay are banned for commercial trawlers, Sığacık Bay is one of the most densely trawled area of the Central Aegean Sea.

The trawl operations were completed between 2008-2009 in Izmir and Sığacık Bay, Central Aegean Sea. One research vessel and a commercial trawler were used during the survey. During the operations, a traditional bottom trawl gear $(48 \mathrm{~mm}$ mesh size in codend) and cover net were used and hauls were limited with 30 minutes and speed was an average of 2.5 knot.

Specimens were identified according to Compagno et al. (2005) and Fricke et al. (2007). The length and weight parameters were measured for statistical analysis. Except for large ones, specimens were brought fresh to the laboratory; abundant ones were put in 30 It barrels with a 4-8 \% concentration of formaldehyde solution and preserved. In laboratory, sex determinations were made macroscopically from outside by the existence of claspers. Total length (TL) and Pre-Supra caudal length (PSCL) measurements were made with a measuring board with sensitivity of $1 \mathrm{~mm}$, and for weight measurements, during trawl operations a scale with sensitivity of $1 \mathrm{~g}$ for very large specimens and those which were brought to the laboratory an electronic scale with a sensitivity of 0.01 were used.
LWR are of power type, i.e., $W=a L^{b}$. In this equation, where $(W)$ is the total weight $(g),(L)$ is the total length $(\mathrm{cm})$, a is the coefficient of body shape, and it gets values around 0.1 for fishes which are small sized and with a rounded body shape, 0.01 for streamlined-shaped fishes and 0.001 for eellike shaped fishes. In contrast, $b$ is the coefficient balancing the dimensions of the equation and its values can be smaller, larger or equal to. In the first two cases (i.e., $b<3$ and $b>3$ ) fish growth is allometric (i.e., when $b<3$ the fish grows faster in length than in weight, and when $b>3$ the fish grows faster in weigth than in length), whereas when $b=3$ growth is isometric (Karachle and Stergiou, 2012).

LWR for species more than 5 individuals, mean lengths and weights, and size ranges were determined and for the species less than 5 individuals their LWRs were not taken into account due to insufficient data but length and weight measurements were given. LWR of species over 5 individuals, except only for Chimaera monstrosa where for $(L)$ PSCL $(\mathrm{cm})$ was used, for $L W R$ parameters for $(L) T L(\mathrm{~cm})$ mesurements were used.

Specimens were divided to male, female and total ( $(+, \hat{\partial}$, and $\Sigma$ ) then; minimum, maximum and average lengths of females, males and total were calculated along with some descriptive statistical parameters and their growth types were identified and tested using Microsoft Office Excel and Statsoft Statistica 7.0 package programs.

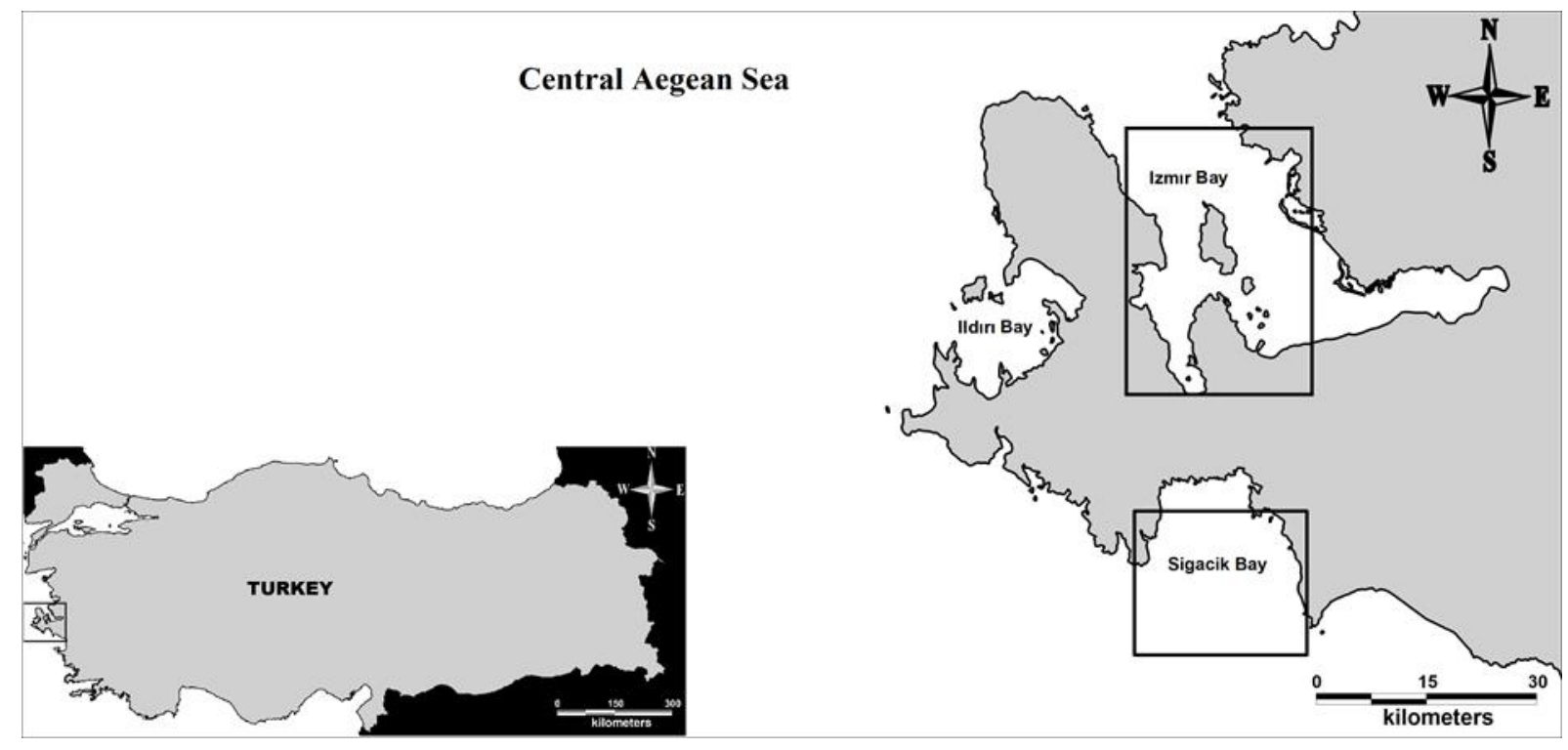

Figure 1. Map showing the study areas. 


\section{RESULTS}

During the study from 2511 individual; 11 Shark species belonging to 3 Order, 8 Family; 18 Batoid species belonging to 2 Order, 5 Family and 1 Chimaera belonging to 1 Order, 1 Family were identified. In total 30 cartilaginous fish species from 6 Orders, 13 Family (from Sığacık Bay 15 species and from Izmir Bay 21 species) were captured.
One of the species (Mustelus punctulatus) LWR parameters were calculated and given in as a first for Turkish waters with the other 15 species in Table 1.

The rest of species, which were under 5 individuals, only their minimum and maximum of the length and weight are shown in Table 2.

Table 1. Length and weight relationships descriptive statistics and parameters of 16 cartilaginous fish species in the central Aegean Sea.

\begin{tabular}{|c|c|c|c|c|c|c|c|c|c|c|c|c|c|c|}
\hline \multirow{2}{*}{ Species } & \multirow{2}{*}{ Mesurment } & \multirow{2}{*}{ Sex } & \multirow{2}{*}{$\mathbf{n}$} & \multicolumn{3}{|c|}{ Length Characteristics (cm) } & \multicolumn{3}{|c|}{ Weight Characteristics (g) } & \multicolumn{4}{|c|}{ Parameters of the Relationship } & \multirow{2}{*}{ Growth Type } \\
\hline & & & & Min & $\operatorname{Max}$ & Mean $\pm \mathbf{S E}$ & Min & Max & Mean $\pm \mathrm{SE}$ & a & B & $\mathbf{R}^{2}$ & SE (b) & \\
\hline \multirow{3}{*}{ Galeus melastomus } & \multirow{3}{*}{$\mathrm{TL}^{*}$} & $\delta$ & 130 & 8,9 & 37,2 & $14.18 \pm 0.344$ & 1,13 & 162,42 & $10.69 \pm 1.747$ & 0,0019 & 3,15 & 0,952 & 0,062 & (+) Allometry \\
\hline & & 9 & 105 & 9,2 & 45 & $14.57 \pm 0.466$ & 1,94 & 278,77 & $13.32 \pm 3.281$ & 0,0019 & 3,14 & 0,949 & 0,072 & (+) Allometry \\
\hline & & $\Sigma$ & 235 & 8,9 & 45 & $14.35 \pm 0.282$ & 1,13 & 278,77 & $11.86 \pm 1.754$ & 0,0019 & 3,14 & 0,95 & 0,047 & (+) Allometry \\
\hline \multirow{3}{*}{ Scyliorhinus canicula } & \multirow{3}{*}{$\mathrm{TL}^{*}$} & 8 & 590 & 7,8 & 51,2 & $20.23 \pm 0.492$ & 0,31 & 458 & $57.37 \pm 4.402$ & 0,0012 & 3,25 & 0,973 & 0,022 & (+) Allometry \\
\hline & & 운 & 620 & 7,8 & 50,9 & $20.18 \pm 0.478$ & 0,63 & 461,66 & $59.67 \pm 4.391$ & 0,0011 & 3,27 & 0,983 & 0,017 & (+) Allometry \\
\hline & & $\Sigma$ & 1210 & 7,8 & 51,2 & $20.21 \pm 0.343$ & 0,31 & 461,66 & $58.55 \pm 3.108$ & 0,0012 & 3,26 & 0,978 & 0,014 & (+) Allometry \\
\hline \multirow{3}{*}{ Scyliorhinus stellaris } & \multirow{3}{*}{$T^{*}$} & 8 & 14 & 25,8 & 69,7 & $50.42 \pm 3.05$ & 60,13 & 1685,6 & $609.99 \pm 136.07$ & 0,0006 & 3,46 & 0,968 & 0,182 & $(+)$ Allometry \\
\hline & & 우 & 5 & 41,6 & 46,6 & $44.56 \pm 0.83$ & 265,84 & 423,26 & $325.13 \pm 29.81$ & 0,0002 & 3,78 & 0,658 & 1,573 & Isometric \\
\hline & & $\Sigma$ & 19 & 25,8 & 69,7 & $48.88 \pm 2.31$ & 60,13 & 1685,6 & $535.03 \pm 103.83$ & 0,0006 & 3,46 & 0,964 & 0,161 & (+) Allometry \\
\hline \multirow{3}{*}{ Mustelus mustelus } & & 8 & 28 & 41,8 & 91,5 & $72.08 \pm 2.81$ & 121,8 & 2690 & $1301.43 \pm 139.15$ & 0,0006 & 3,39 & 0,981 & 0,093 & (+) Allometry \\
\hline & $T^{*}$ & 오 & 13 & 42 & 113,3 & $72.74 \pm 6.50$ & 190 & 4780 & $1732.76 \pm 431.54$ & 0,0017 & 3,16 & 0,971 & 0,164 & Isometric \\
\hline & & $\sum$ & 41 & 41,8 & 113,3 & $72.29 \pm 2.77$ & 121,8 & 4780 & $1438.19 \pm 166.28$ & 0,001 & 3,27 & 0,971 & 0,091 & (+) Allometry \\
\hline & & 8 & 5 & 70,9 & 89,3 & $82.64 \pm 3.34$ & 1104 & 2244 & $1738.14 \pm 212.15$ & 0,0026 & 3,03 & 0,954 & 0,383 & Isometric \\
\hline Mustelus punctulatus & $\mathrm{TL}^{*}$ & 오 & 1 & - & - & 55 & - & - & 451,91 & - & - & - & - & \\
\hline & & $\sum$ & 6 & 55 & 89,3 & $78.03 \pm 5.35$ & 451,91 & 2244 & $1523.77 \pm 275.60$ & 0,0012 & 3,21 & 0,991 & 0,157 & Isometric \\
\hline & & 8 & 62 & 8,9 & 26,4 & $16.73 \pm 0.591$ & 2,71 & 81,01 & $25.78 \pm 2.713$ & 0,0031 & 3,12 & 0,981 & 0,056 & (+) Allometry \\
\hline Etmopterus spincx & TL* & 우 & 67 & 8,6 & 31,7 & $17.52 \pm 0.645$ & 2,2 & 150,81 & $30.08+3.434$ & 0,0039 & 3,04 & 0,978 & 0,056 & Isometric \\
\hline & & $\sum$ & 129 & 8,6 & 31,7 & $17.14 \pm 0.439$ & 2,2 & 150,81 & $28.01 \pm 2.209$ & 0,0035 & 3,08 & 0,98 & 0,04 & (+) Allometry \\
\hline & & 8 & 149 & 17,8 & 60 & $27.22 \pm 0.867$ & 16 & 881,18 & $127.78 \pm 15.641$ & 0,0049 & 2,95 & 0,963 & 0,048 & Isometric \\
\hline Squalus blainvillei & $\mathrm{TL}^{*}$ & 우 & 159 & 16,2 & 70,5 & $26.15 \pm 0.878$ & 5,18 & 1587,37 & $133.91 \pm 20.516$ & 0,0046 & 2,98 & 0,94 & 0,06 & metric \\
\hline & & $\sum$ & 308 & 16,2 & 70,5 & $26.67 \pm 0.617$ & 5,18 & 1587,37 & $130.94 \pm 12.997$ & 0,0048 & 2,96 & 0,95 & 0,039 & Isometric \\
\hline & & 8 & 48 & 10 & 27,9 & $16.10 \pm 0.68$ & 24,57 & 413,29 & $104.54 \pm 13.77$ & 0,0365 & 2,79 & 0,985 & 0,051 & (-) Allometry \\
\hline orpedo marmorata & $T^{*}$ & 오 & 59 & 9,6 & 39,3 & $18.92 \pm 1.075$ & 7,98 & 1310,42 & $235.36 \pm 43.444$ & 0,0188 & 3,02 & 0,985 & 0,102 & Isometric \\
\hline & & $\sum$ & 107 & 9,6 & 39,3 & $17.66 \pm 0.677$ & 7,98 & 1310,42 & $176.68 \pm 25.44$ & 0,023 & 2,96 & 0,939 & 0,066 & Isometric \\
\hline & & 8 & 4 & 19 & 46,5 & 31,38 & 14,78 & 285,04 & 118,5 & 0,0038 & - & - & - & \\
\hline Dipturus oxyrinchus & $\mathrm{TL}^{*}$ & 웅 & 4 & 18,1 & 44,5 & 29,9 & 15,37 & 227,11 & 103,68 & 0,0039 & - & - & - & \\
\hline & & $\sum$ & 8 & 18,1 & 46,5 & $30.64 \pm 4.286$ & 14,78 & 285,04 & $111.09 \pm 37.816$ & 0,0309 & 3,13 & 0,995 & 0,093 & Isometric \\
\hline & & 8 & 8 & 9,7 & 22,3 & $12.7 \pm 1.52$ & 19,41 & 218 & $58.09 \pm 24.15$ & 0,0276 & 2,9 & 0,992 & 0,109 & Isometric \\
\hline Torpedo nobiliana & $\mathrm{TL}^{*}$ & 9 & 2 & 11,9 & 13,6 & 12,75 & 35,13 & 47,99 & 41,56 & - & - & - & - & \\
\hline & & $\sum$ & 10 & 9,7 & 22,3 & $12.71 \pm 1.206$ & 19,41 & 218 & $54.79 \pm 19.201$ & 0,0284 & 2,89 & 0,989 & 0,108 & Isometric \\
\hline & & $\delta$ & 9 & 18,5 & 33 & \pm 1.755 & 20,1 & 143,15 & .788 & 0,0008 & 3,46 & 0,992 & 0,116 & Allometry \\
\hline Raja asterias & $\mathrm{TL}^{*}$ & 9 & 8 & 19 & 41,5 & $30.84 \pm 2.289$ & 19,28 & 311,5 & $124.82 \pm 30.558$ & 0,0006 & 3,52 & 0,996 & 0,067 & (+) Allometry \\
\hline & & $\sum$ & 17 & 18,5 & 41,5 & $28.34 \pm 1.497$ & 19,28 & 311,5 & $96.01 \pm 17.212$ & 0,0007 & 3,47 & 0,994 & 0,097 & (+) Allometry \\
\hline & & $\delta$ & 59 & 12,7 & 60,5 & $28.26 \pm 1.357$ & 5,97 & 1200 & $140.2 \pm 26.443$ & 0,0007 & 3,5 & 0,974 & 0,044 & (+) Allometry \\
\hline Raja clavata & $\mathrm{TL}^{*}$ & 9 & 78 & 12,6 & 70,2 & $30.19 \pm 1.319$ & 6,01 & 2160 & $192.83 \pm 38.396$ & 0,0007 & 3,48 & 0,991 & 0,079 & (+) Allometry \\
\hline & & $\Sigma$ & 137 & 12,6 & 70,2 & $29.4 \pm 0.951$ & 5,97 & 2160 & $170.16 \pm 24.671$ & 0,0006 & 3,52 & 0,963 & 0,049 & (+) Allometry \\
\hline & & $\delta$ & 6 & 41,5 & 57,4 & $51.9 \pm 2.439$ & 418,58 & 1160 & $891.43 \pm 118.897$ & 0,0035 & 3,14 & 0,959 & 0,335 & Isometric \\
\hline Raja radula & $\mathrm{TL}^{*}$ & 9 & 10 & 43,2 & 61,2 & $54.03 \pm 1.696$ & 480 & 1604 & $1115.41 \pm 107.263$ & 0,0018 & 3,33 & 0,946 & 0,281 & Isometric \\
\hline & & $\sum$ & 16 & 41,5 & 61,2 & $53.23 \pm 1.376$ & 418,58 & 1604 & $1031.42 \pm 82.858$ & 0,0017 & 3,33 & 0,94 & 0,226 & Isometric \\
\hline & & $\delta$ & 4 & 29,3 & 33 & $30.7 \pm 0.871$ & 118,56 & 170,11 & $140.2 \pm 11.063$ & - & - & - & - & \\
\hline Rostroraja alba & $\mathrm{TL}^{*}$ & 9 & 6 & 25,1 & 124 & $51.92 \pm 15.238$ & 74 & 15000 & $2804.29 \pm 2444.288$ & 0,0014 & 3,35 & 0,997 & 0,087 & (+) Allometry \\
\hline & & $\Sigma$ & 10 & 25,1 & 124 & $43.43 \pm 9.46$ & 74 & 15000 & $1738.65 \pm 1476.751$ & 0,0016 & 3,32 & 0,997 & 0,063 & (+) Allometry \\
\hline & & $\delta$ & 36 & 36,5 & 80 & $57.01 \pm 1.806$ & 295,14 & 4000 & $1438.13 \pm 147.36$ & 0,0021 & 3,29 & 0,954 & 0,124 & (+) Allometry \\
\hline Dasyatis pastinaca & $\mathrm{TL}^{*}$ & 9 & 42 & 33,4 & 138 & $64.77 \pm 3.519$ & 191,38 & 21100 & $3309.24 \pm 713.501$ & 0,9713 & 3,51 & 0,971 & 0,095 & (+) Allometry \\
\hline & & $\sum$ & 78 & 33,4 & 138 & $61.19 \pm 2.104$ & 191,38 & 21100 & $2445.65 \pm 402.264$ & 0,0011 & 3,46 & 0,968 & 0,072 & (+) Allometry \\
\hline & & $\delta$ & 14 & 41,1 & 87,5 & $60.09 \pm 4.47$ & 67,65 & 2260 & $897.32 \pm 205.793$ & 0,0009 & 3,29 & 0,776 & 0,51 & Isometric \\
\hline yliobatis aquila & $T L^{*}$ & 오 & 40 & 43,5 & 179,5 & $96.27 \pm 5.656$ & 168,6 & 15800 & $4783 \pm 715.256$ & 0,0004 & 3,5 & 0,963 & 0,111 & (+) Allometry \\
\hline & & $\sum$ & 54 & 41,1 & 179,5 & $86.89 \pm 4.843$ & 67,65 & 15800 & $3775.6 \pm 579.877$ & 0,0005 & 3,42 & 0,946 & 0,114 & (+) Allometry \\
\hline & & $\delta$ & 40 & 7,8 & 43,3 & $14.73 \pm 1.195$ & 4,9 & 557,6 & $53.56 \pm 18.413$ & 0,0108 & 2,89 & 0,969 & 0,084 & Isometric \\
\hline Chimaera monstrosa & $\mathrm{PSCL}^{* *}$ & 9 & 57 & 8,2 & 45,5 & $14.69 \pm 1.154$ & 4,79 & 1038,2 & $79.77 \pm 28.388$ & 0,0062 & 3,11 & 0,984 & 0,053 & (+) Allometry \\
\hline & & $\sum$ & 97 & 7,8 & 45,5 & $14.71 \pm 0.834$ & 4,79 & 1038,2 & $68.96 \pm 18.297$ & 0,0076 & 3,03 & 0,978 & 0,047 & Isometric \\
\hline
\end{tabular}


Table 2. Length and weight measurements of 14 cartilaginous fish species, which were less than 5 individuals

\begin{tabular}{|c|c|c|c|c|c|c|}
\hline \multirow[t]{2}{*}{ Species } & \multirow[t]{2}{*}{ Sex } & \multirow[t]{2}{*}{$\mathbf{n}$} & \multicolumn{2}{|c|}{$\begin{array}{c}\text { Length } \\
\text { Characteristics (cm) }\end{array}$} & \multicolumn{2}{|c|}{$\begin{array}{c}\text { Weight } \\
\text { Characteristics (g) }\end{array}$} \\
\hline & & & Min & Max & Min & Max \\
\hline $\begin{array}{l}\text { Heptranchias } \\
\text { perlo }\end{array}$ & 우 & 1 & \multicolumn{2}{|c|}{99.6} & \multicolumn{2}{|c|}{4382} \\
\hline $\begin{array}{l}\text { Galeorhinus } \\
\text { galeus }\end{array}$ & q & 1 & \multicolumn{2}{|c|}{99.8} & \multicolumn{2}{|c|}{3340} \\
\hline \multirow{3}{*}{$\begin{array}{l}\text { Mustelus } \\
\text { punctulatus }\end{array}$} & $0^{\pi}$ & 5 & 70.9 & 89.3 & 7.47 & 1104 \\
\hline & 우 & 1 & \multicolumn{2}{|c|}{55} & \multicolumn{2}{|c|}{451.91} \\
\hline & $\sum$ & 6 & 55 & 89.3 & 13.12 & 451.91 \\
\hline \multirow{3}{*}{$\begin{array}{l}\text { Oxynotus } \\
\text { centrina }\end{array}$} & $\hat{0}$ & 1 & \multicolumn{2}{|c|}{56.3} & \multicolumn{2}{|c|}{1180.84} \\
\hline & q & 1 & \multicolumn{2}{|c|}{61.5} & \multicolumn{2}{|c|}{2845.36} \\
\hline & 0 & 2 & 39 & 54.7 & 284.62 & 786.39 \\
\hline \multirow[t]{2}{*}{ Dalatias licha } & 우 & 3 & 32.1 & 36.6 & 151.68 & 195.69 \\
\hline & $\sum$ & 5 & 32.1 & 54.7 & 151.68 & 786.39 \\
\hline \multirow{2}{*}{ Dipturus batis } & $\hat{0}$ & 1 & \multicolumn{2}{|c|}{47} & \multicolumn{2}{|c|}{880.26} \\
\hline & q & 1 & \multicolumn{2}{|c|}{56.1} & \multicolumn{2}{|c|}{3125.75} \\
\hline \multirow{3}{*}{$\begin{array}{l}\text { Leucoraja } \\
\text { fullonica }\end{array}$} & $\hat{\sigma}$ & 2 & 9.3 & 34.6 & 24.98 & 144.75 \\
\hline & P & 1 & \multicolumn{2}{|c|}{28} & \multicolumn{2}{|c|}{74.95} \\
\hline & $\sum$ & 3 & 9.3 & 34.6 & 24.98 & 144.75 \\
\hline \multirow{3}{*}{$\begin{array}{l}\text { Leucoraja } \\
\text { naevus }\end{array}$} & 0 & 2 & 51.2 & 58 & 791.14 & 985.42 \\
\hline & q & 1 & \multicolumn{2}{|c|}{62} & \multicolumn{2}{|c|}{1497.32} \\
\hline & $\sum$ & 3 & 51.2 & 62 & 791.14 & 1497.32 \\
\hline \multirow{2}{*}{ Raja miraletus } & $\hat{\sigma}$ & 1 & \multicolumn{2}{|c|}{21.2} & \multicolumn{2}{|c|}{129.03} \\
\hline & q & 1 & & & & \\
\hline Raja montagui & q & 1 & & & & \\
\hline $\begin{array}{l}\text { Raja } \\
\text { polystigma }\end{array}$ & 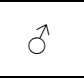 & 2 & 53.9 & 55.5 & 902.59 & 979.41 \\
\hline $\begin{array}{l}\text { Dasyatis } \\
\text { tortonesei }\end{array}$ & 우 & 1 & & & & \\
\hline $\begin{array}{l}\text { Gymnura } \\
\text { altavela }\end{array}$ & q & 4 & 33 & 43.8 & 949.44 & 2005.32 \\
\hline $\begin{array}{l}\text { Pteromylaeus } \\
\text { bovinus }\end{array}$ & 우 & 2 & 73 & 106 & 720.84 & 2364.68 \\
\hline
\end{tabular}

The most abundant species were Scyliorhinus canicula $(n=1210)$, Squalus blainvillei $(n=308)$, Galeus melastomus $(n=235)$. In total the TL ranged from 7.8 (Scyliorhinus canicula) to $179.5 \mathrm{~cm}$ (Myliobatis aquila). The values of the slope b ranged from 2.791 (Torpedo marmorata) to 3.778 (Scyliorhinus stellaris). The a values ranged from 0.0002 (Scyliorhinus stellaris) to 0.226 (Raja radula).

Growth type according to $b$ values of both sexes was identified as (+) allometry for 3 shark, 4 batoid, while 1 shark and 2 batoid was identified as isometric. Additionally, growth type difference was observed between sexes of 3 sharks, 4 batoid and 1 chimaera species. But when these differences were tested with ANCOVA it resulted as there were no growth type difference between sexes of Torpedo marmorata, Dasyatis pastinaca (2 Batoid) and Chimaera monstrosa.

\section{DISCUSSION}

The other studies conducted in study areas (Izmir Bay and Sığacık Bay) mostly focus on bony fish of the areas but few have given some information on cartilaginous fish species. This study was compared with six studies from northern Aegean, three studies from central Aegean, four studies from Mediterranean and one from Black Sea and one from the Sea of Marmara. The LWR estimations and growth types for some of the other studies are given in Table 3. 
Table 3. Length (L)-weight (W) relationships comparison with the studies from Turkish seas. *B: Black Sea; CA: Central Aegean; NA: Northern Aegean; M: Marmara; MED: Mediterranean. GT: growth type, + A: positive allometry, l: isometric, - A: negative allometry.

\begin{tabular}{|c|c|c|c|c|c|c|c|c|}
\hline \multirow{2}{*}{ Species } & \multirow{2}{*}{$\mathbf{n}$} & \multirow{2}{*}{ W-L } & \multirow{2}{*}{ GT } & \multirow{2}{*}{ Authors } & \multirow{2}{*}{ Study Areas* } & \multicolumn{3}{|c|}{ Present Work } \\
\hline & & & & & & $\mathbf{n}$ & W-L & GT \\
\hline \multirow{9}{*}{ Raja clavata } & 29 & $\mathrm{~W}=0.0016 \mathrm{~L}^{3.29}$ & $+\mathrm{A}$ & Filiz and Mater 2002 & NA-CA & \multirow{9}{*}{137} & \multirow{9}{*}{$\mathrm{W}=0.0007 \mathrm{~L}^{3.50}$} & \multirow{9}{*}{$+\mathrm{A}$} \\
\hline & 37 & $\mathrm{~W}=0.0016 \mathrm{~L}^{3.30}$ & & Filiz and Bilge 2004 & $\mathrm{CA}$ & & & \\
\hline & 32 & $\mathrm{~W}=0.0322 \mathrm{~L}^{2.60}$ & I & Yarmaz 2009 & NA & & & \\
\hline & 112 & $\mathrm{~W}=0.0132 \mathrm{~L}^{3.12}$ & & Ismen et. al. 2007 & NA & & & \\
\hline & 226 & $\mathrm{~W}=0.00163 \mathrm{~L}^{3.32}$ & & Yigin and Ismen 2008 & NA & & & \\
\hline & 77 & $\mathrm{~W}=0.0037 \mathrm{~L}^{3.08}$ & & Yeldan and Avşar 2007 & MED & & & \\
\hline & 75 & $\mathrm{~W}=0.023 \mathrm{~L}^{2.64}$ & & Basusta et. al. 2012 & MED & & & \\
\hline & 792 & $\mathrm{~W}=0.0018 \mathrm{~L}^{3.26}$ & $+\mathrm{A}$ & Saygu 2011 & MED & & & \\
\hline & 27 & $\mathrm{~W}=0.0019 \mathrm{~L}^{3.24}$ & $+\mathrm{A}$ & Demirhan and Can 2007 & $\mathrm{~B}$ & & & \\
\hline \multirow{6}{*}{ Raja radula } & 23 & $\mathrm{~W}=0.0029 \mathrm{~L}^{3.21}$ & $\bar{I}$ & Yarmaz 2009 & $\mathrm{NA}$ & \multirow{6}{*}{16} & \multirow{6}{*}{$\mathrm{W}=0.0017 \mathrm{~L}^{3.33}$} & \multirow{6}{*}{ I } \\
\hline & 25 & $\mathrm{~W}=0.0030 \mathrm{~L}^{3.21}$ & & Karakulak et. al. 2006 & NA & & & \\
\hline & 49 & $\mathrm{~W}=0.01131 \mathrm{~L}^{3.25}$ & & Ismen et. al. 2007 & NA & & & \\
\hline & 204 & $\mathrm{~W}=0.00205 \mathrm{~L}^{3.32}$ & & Yigin and Ismen 2008 & NA & & & \\
\hline & 295 & $\mathrm{~W}=0.00121 \mathrm{~L}^{3.36}$ & & Yeldan and Avşar 2007 & MED & & & \\
\hline & 62 & $\mathrm{~W}=0.0174 \mathrm{~L}^{3.07}$ & $+\mathrm{A}$ & Saygu 2011 & MED & & & \\
\hline \multirow{3}{*}{ Rostroraja alba } & 11 & $\mathrm{~W}=0.009 \mathrm{~L}^{3.49}$ & & Ozaydın et al. 2007 & $\mathrm{CA}$ & \multirow{3}{*}{10} & \multirow{3}{*}{$\mathrm{W}=0.0016 \mathrm{~L}^{3.32}$} & \multirow{3}{*}{$+\mathrm{A}$} \\
\hline & 43 & $\mathrm{~W}=0.00662 \mathrm{~L}^{3.20}$ & & Ismen et. al. 2007 & NA & & & \\
\hline & 126 & $\mathrm{~W}=0.00194 \mathrm{~L}^{3.27}$ & & Yigin and Ismen 2008 & NA & & & \\
\hline \multirow{7}{*}{ Scyliorhinus canicula } & 110 & $\mathrm{~W}=0.0016 \mathrm{~L}^{3.18}$ & $+\mathrm{A}$ & Filiz and Mater, 2002 & $\mathrm{NA}-\mathrm{CA}$ & \multirow{7}{*}{1210} & \multirow{7}{*}{$\mathrm{W}=0.0012 \mathrm{~L}^{3.26}$} & \multirow{7}{*}{$+\mathrm{A}$} \\
\hline & 637 & $\mathrm{~W}=0.0012 \mathrm{~L}^{3.26}$ & & Filiz and Bilge 2004 & $\mathrm{CA}$ & & & \\
\hline & 187 & $\mathrm{~W}=0.0006 \mathrm{~L}^{3.44}$ & & Ozaydin et al. 2007 & $\mathrm{CA}$ & & & \\
\hline & 1501 & $\mathrm{~W}=0.00169 \mathrm{~L}^{3.17}$ & & Ismen et. al. 2007 & NA & & & \\
\hline & 1888 & $\mathrm{~W}=0.0017 \mathrm{~L}^{3.17}$ & & Ismen et. al. 2009 & NA & & & \\
\hline & 108 & $\mathrm{~W}=8 \mathrm{E}-06 \mathrm{~L}^{2.88}$ & I & Yarmaz 2009 & NA & & & \\
\hline & 189 & $\mathrm{~W}=0.004 \mathrm{~L}^{2.87}$ & & Demirel and Dalkara 2012 & $\mathrm{M}$ & & & \\
\hline Scyliorhinus stellaris & 12 & $\mathrm{~W}=0.0009 \mathrm{~L}^{3.37}$ & & Ismen et. al. 2009 & $\mathrm{NA}$ & 19 & $\mathrm{~W}=0.0006 \mathrm{~L}^{3.46}$ & $+\mathrm{A}$ \\
\hline \multirow{4}{*}{ Squalus blainvillei } & 299 & $\mathrm{~W}=0.00345 \mathrm{~L}^{3.06}$ & & Ismen et. al. 2007 & NA & \multirow{4}{*}{308} & & \\
\hline & 27 & $\mathrm{~W}=0.0030 \mathrm{~L}^{3.07}$ & & Ismen et. al. 2009 & NA & & $\mathrm{W}=00048 \mathrm{I}^{2.96}$ & I \\
\hline & 22 & $\mathrm{~W}=0.0139 \mathrm{~L}^{3.10}$ & & Karakulak et. al. 2006 & NA & & $W=0.0048 \mathrm{~L}$ & 1 \\
\hline & 20 & $\mathrm{~W}=0.05920 \mathrm{~L}^{2.64}$ & & Ismen et. al. 2007 & NA & & & \\
\hline & 20 & $\mathrm{~W}=0.0488 \mathrm{~L}^{2.69}$ & $-\mathrm{A}$ & Filiz and Mater 2002 & NA-CA & & & \\
\hline & 37 & $\mathrm{~W}=0.0273 \mathrm{~L}^{2.91}$ & & Filiz and Bilge 2004 & $\mathrm{CA}$ & & & \\
\hline Tornedo marmorata & 12 & $\mathrm{~W}=0.0535 \mathrm{~L}^{2.39}$ & & Ozaydin et al. 2007 & $\mathrm{CA}$ & 107 & W-0 2.95 & $\mathrm{~J}$ \\
\hline 10rpeao marmorata & 9 & $\mathrm{~W}=0.1297 \mathrm{~L}^{2.47}$ & I & Yarmaz 2009 & NA & 107 & $W=0.023 \mathrm{~L}$ & 1 \\
\hline & 22 & $\mathrm{~W}=0.0139 \mathrm{~L}^{3.10}$ & & Karakulak et. al. 2006 & NA & & & \\
\hline & 20 & $\mathrm{~W}=0.05920 \mathrm{~L}^{2.64}$ & & Ismen et. al. 2007 & NA & & & \\
\hline Torpedo nobiliana & 92 & $\mathrm{~W}=0.015 \mathrm{~L}^{3.06}$ & & Basusta et. al. 2012 & MED & 10 & $\mathrm{~W}=0.028 \mathrm{~L}^{2.89}$ & $\mathrm{I}$ \\
\hline
\end{tabular}


The differences between LWR estimations of the other studies may caused by, especially for different regions, to ecological differences of the sampling areas. Also may be the result of lack or abundance of food, specimens age, reproductive stage (immature, maturing or mature), season of the sampling, differences between sampling depth and also to the fishing gears selectivity such as longlines could only sample specimens over some size when trawl can sample more diversely sized samples. On the subject of growth type there were difference with the result of this study and with Bilge et al. (2010), Demirhan and Can (2007), Filiz and Mater (2002), Ismen (2003), Saygu (2011) and Yarmaz (2009) studies which were conducted in Central Aegean Sea, Black Sea, North and Central Aegean Sea, Mediterranean Sea, Mediterranean Sea and North Aegean Sea, respectively. In general there were difference between sampling methodology (fishing gear and sampling season) but the differences can be the result of some other reasons listed in previous sentences.

In general, the results were similar to previous studies in other countries but there were few differences may have caused by insufficient number of species. A study from western Mediterranean by Merella et al. (1997) estimated Scyliorhinus canicula ( $\mathrm{n}=262)$, Squalus blainvillei $(\mathrm{n}=27)$, Raja asterias $(n=11)$ and Raja clavata $(n=18)$ species (a) values were $0.0016,0.0012,0.0018$ and 0.0024 , and (b) values were $3.16,3.37,3.27$ and 3.20 , respectively. The results in this study were close (Scyliorhinus canicula $n=1210$

\section{REFERENCES}

Bilecenoglu, M., Kaya, M., Cihangir, B., Cicek, E., 2014. An Updated Checklist of The Marine Fishes of Turkey. Turkish Journal of Zoology. 38. doi:10.3906/zoo-1405-60

Bilge, G., Filiz, H., Tarkan, A. N., 2010. Length-Weight Relationship of Velve Belly Lantern Shark Etmopterus spinax (Linnaeus, 1758) in Sigacik bay (Aegean Sea). Istanbul University Journal of Fisheries and Aquatic Sciences, $25(1): 1-8$.

Basusta, A., Basusta, N., Sulikowski, J. A., Driggers, W. B., Demirhan, S. A., Cicek, E., 2012. Length-weight relationships for nine species of batoids from the Iskenderun Bay, Turkey. Journal of Applied Ichthyology, 28 850-851. doi:10.1111/j.1439-0426.2012.02013.x

Bradai M.N., Saidi B., Enajjar S., 2012. Elasmobranchs of the Mediterranean and Black sea: status, ecology and biology. Bibliographic analysis Studies and Reviews. General Fisheries Commission for the Mediterranean, No. 91. Rome, FAO. 103 pp.

Compagno, L., Dando, M., Fowler, S., 2005. Sharks of the World. Princeton Field Guides.

Demirel, N., Dalkara, M., 2012. Weight-length relationships of 28 fish species in the Sea of Marmara. Turkish Journal of Zoology, 36(6): 785-791. doi:10.3906/zoo-1111-29

Demirhan, S. A., Can, M. F., 2007. Length-weight relationships for seven fish species from the southeastern Black Sea. Journal of Applied Ichthyology 23, 282-283. doi: 10.1111/j.1439-0426.2007.00835.x

Eronat, E., G., T., Ozaydin, O., 2014. Diet composition of the Thornback Ray, Raja clavata Linnaeus, 1758 (Elasmobranchii: Rajidae) in the Turkish Aegean Sea, Zoology in the Middle East. doi: 10.1080/09397140.2014.994312.
$\mathrm{W}=0.0012 \mathrm{~L}^{3.26}$, Squalus blainvillei $\mathrm{n}=308 \mathrm{~W}=0.0048 \mathrm{~L}^{2.96}$, Raja asterias $\mathrm{n}=17 \mathrm{~W}=0.0007 \mathrm{~L}^{3.48}$ and Raja clavata $\mathrm{n}=187$ $\mathrm{W}=0.0005 \mathrm{~L}^{3.61}$ ). When comparing with a study from southern Portugal by Santos et al. (2002); estimated parameters (a) and (b) for species Galeus melastomus and Scyliorhinus canicula as 0.0022 and $3.056,0.0017$ and 3.180 , respectively which were close to the findings of Galeus melastomus (0.0019 and 3.14) and Scyliorhinus canicula (0.0012 and 3.26) species from this study. Another study from eastern-central Atlantic estimated parameter (a) for species Galeus melastomus ( $\mathrm{n}=98)$ as 0.00145 and for Mustelus mustelus $(\mathrm{n}=14)$ as 0.00162 and parameter (b) for Galeus melastomus as 3.161 and for Mustelus mustelus as 3.178 (Ferreira et al. 2008). Ferreira et al. (2008) also estimated the species parameter (a) by setting (b) to 3 because of insufficient number of Rostroraja alba (2 specimens) as 0.00638 . When compared with this study the findings were also close but differed as a result of more sufficient sampling in this study (Galeus melastomus $\mathrm{n}=235$, $\mathrm{a}=0.0019$ and $\mathrm{b}=3.14$; Mustelus mustelus $n=41, a=0.001$ and $b=3.27$; Rostroraja alba $n=10$, $a=0.0016$ and $b=3.32$ ).

In conclusion; this study obtained and examined the most chondrichthyan species (11 Sharks, 18 Batoids and 1 Chimaera) among the studies in the eastern Mediterranean and estimated LWR for 16 species. This study will be a valuable resource for future studies on the subject and for the study areas.

Ferreira, S., Sousa, R., Delgado, J., Carvalho, D., Chada, T., 2008. Weightlength relationships for demersal fish species caught off the Madeira archipelago (eastern-central Atlantic. Journal of Applied Ichthyology, 24, 93-95. doi: 10.1111/j.1439-0426.2007.01027.x.

Filiz, H., Mater, S., 2002. A Preliminary Study on Length-Weight Relationships for Seven Elasmobranch Species from North Aegean Sea, Turkey. Ege $J$ Fish Aqu Sci 19: 401-409.

Filiz, H., Bilge, G., 2004. Length-weight relationships of 24 fish species from the North Aegean Sea, Turkey. Journal of Applied Ichthyology, 20, 431$432 \mathrm{pp}$.

Filiz, H., Taşkavak, E., 2006. Sexual dimorphism in the head, mouth and body morphology ofthe smallspotted catshark, Scyliorhinuscanicula (Linnaeus, 1758) (Chondrichthyes: Scyliorhinidae) from Turkey. Acta Adriatica. 47(1), 37-47.

Fricke, R., Bilecenoglu, M., Sari, H. S., 2007. Annotated checklist of fish and lamprey species (Gnathostomata and Petromyzontomorphi) of Turkey, including a red list of threatened and declining species. Stuttgarter Beitr. Naturk. Ser. A Nr. 706, 169 p.

Ismen, A., 2003. Age, growth, reproduction and food of common stingray (Dasyatis pastinaca L., 1758) in Iskenderun Bay, the eastern Mediterranean. Fisheries Research 60: 169-176. doi: 10.1016/S0165-7836(02)00058-9

Ismen, A., Ozen, A., Altinagac, U., Ozekinci, U., Ayaz, A., 2007. Weightlength relationships of 63 fish species in Saros Bay, Turkey. Journal of Applied Ichthyology 23: 707-708 doi: 10.1111/j.1439-0426.2007.00872.x. 
Ismen, A., Yigin n, C. C., Altinagac, U., Ayaz, A., 2009. Length-weigh relationships for ten shark species from Saros Bay (North Aegean Sea). Journal of Applied Ichthyology, 25: 109-112. doi: 10.1111/j.1439-0426.2009.01263.x.

Karachle, P.K., Stergiou K.I., 2012. Morphometrics and allometry in fishes. p. 65-86. In: Morphometrics. Wahl, C (Ed.). In Tech. Available from: http://www.intechopen.com/articles/show/title/morphometrics-andallometry-in-fishes. ISBN: 978-953-51-0172-7.

Karakulak, F. S., Erk, H., Bilgin, B., 2006. Length-weight relationships for 47 coastal fish species from the northern Aegean Sea, Turkey. Journal of Applied Ichthyology, 22: 274-278. doi: 10.1111/j.1439-0426.2006.00736.x.

Kolher, N., Casey, J., Turner, P., 1995. Length-weight relationships for 13 species of sharks from the western North Atlantic. Fishery Bulletin, 93 412-418.

Merella, P., Quetglas, A., Alemany, F., Carbonell, A., 1997. Length-weight relationship of fishes and cephalopods from the Balearic Islands (Western Mediterranean). Naga, The ICLARM Quarterly, 20(3-4), pp. 6668.

Ozaydin, O., Uçkun, D., Akalın, S., Leblebici, S., Tosunoglu, Z., 2007. Length-weight relationships of fishes captured from Izmir Bay, Central Aegean Sea. Journal of Applied Ichthyology, 23, 695-696 p.

Pauly, D., 1993. Fishbyte section editorial. Naga ICLARM, Q. 16, 26.

Saglam, H., Ak, O., 2012. Reproductive biology of Raja clavata (Elasmobranchii: Rajidae) from Southern Black Sea coast around Turkey. Helgoland Marine Research, 66:117-126. doi: 10.1007/s10152-011-0252-5.
Santos, M. N., Gaspar, M. B., Vasconcelos, P., Monteiro, C. C., 2002. Weight-length relationships for 50 selected fish species of the Algarve coast (southern Portugal). Fisheries Research, 59: 289-295. doi: 10.1016/S0165-7836(01)00401-5.

Saygu, I., 2011. Antalya Körfezi Dip Trol Balıkçılığındada Hedef Dışı Avlanan Vatoz Balıkları ve Sağ Kalma Oranlarının Belirlenmesi. Akdeniz University, Faculty of Fisheries. Master Thesis.

Yarmaz, A., 2009. Edremit Körfezi ve Civarında Yaşayan Kıkırdakıı Balıklar ve Bazı Türlerin Biyolojik Özellikleri. Balıkesir University, Institute of Science, Department of Biology. Master Thesis.

Yeldan, H., Avşar, D., 2007. Length-weight relationship for five elasmobranch species from the Cilician Basin shelf waters (Northeastern Mediterranean). Journal of Applied Ichthyology, 23: 713-714. doi: 10.1111/j.1439-0426.2007.00858.x.

Yigin, C. C., Ismen, A., 2008. Length-weight relationships for seven rays from Saros Bay (North Aegean Sea). Journal of Applied Ichthyology, 25:106108. doi: 10.1111/j.1439-0426.2008.01161.x.

Yigin, C. C, Ismen, A., 2010a. Diet of Thornback Ray (Raja clavata Linnaeus, 1758) in Saros Bay (The North Aegean Sea). Rapp.Comm.Int. Mer. Medit. (CIESM), 39.

Yigin, C. C, Işmen, A., 2010b. Age, growth, reproduction and feed of longnosed skate, Dipturus oxyrinchus (Linnaeus, 1758) in Saros Bay, the north Aegean Sea Journal of Applied Ichthyology, 26 913-919. doi: 10.1111/j.1439-0426.2010.01510.x. 